\title{
Retornos médios à educação nos estados brasileiros: uma abordagem com Dados em Painel para as rendas domiciliares per capita ${ }^{1}$
}

\author{
Henrique Viana Espinosa de Oliveira* \\ Ricardo Ramalhete Moreira**
}

\begin{abstract}
Resumo
Este trabalho aborda os efeitos da educação nos rendimentos dos domicílios dos estados brasileiros em termos per capita. As estimações com Dados Empilhados e Dados em Painel foram feitas com dados disponíveis para os anos de 1998 a 2007, poe meio da abordagem de Mincer (1974), com inclusão de variáveis de controle e uma proxy para a experiência de trabalho. As duas principais evidências do trabalho são: a) a elevação de um ano adicional de escolaridade vem acompanhada de retorno de 13,45\% na renda domiciliar per capita dos estados brasileiros; b) políticas estruturais de controle da informalidade parecem ser mais eficazes do que políticas de controle da taxa de desemprego, no que diz respeito aos seus efeitos sobre a renda das famílias brasileiras.
\end{abstract}

Palavras-chave: Retornos à educação. Estados brasileiros. Dados em Painel.

* Bacharel em Ciências Econômicas pela UFES. E-mail: henriqueespinosa.mv@gmail.com

*** Professor no Programa de Pós-Graduação em Economia da UFES. E-mail: ramalhete.s@gmail.com

http://dx.doi.org/10.5335/rtee.v20i43.4597

SUBMISSÃO: 08/05/2014. ACEITE: 21/08/2014. 


\section{Introdução}

De acordo com Salvato e Silva (2008), a importância da educação consiste basicamente na aquisição de mais conhecimento, o que eleva a capacidade de raciocínio do indivíduo, fazendo com que esse seja mais eficiente ao executar as atividades a ele concedidas. Muitos são os fatores que determinam a remuneração de um trabalhador, sendo talvez o principal deles a educação, hoje considerada peça fundamental na formação do capital humano e responsável por uma nova perspectiva para as teorias de crescimento econômico (JONES; ROMER, 2010).

Não é novidade que investimento em educação é algo fundamental para o desenvolvimento de um país, fato que é comprovado pelos inúmeros debates que estão sempre em pauta. Neste contexto, vale lembrar que uma das revindicações feitas nos protestos de junho e julho de 2013, no Brasil, era o pedido de $10 \%$ do valor do Produto Interno Bruto (PIB) a ser investido na educação, enquanto esse valor seria, à época, de 6,1\% do PIB. (ALACÂNTARA, 2013). Os desafios do Brasil nos próximos anos, dentre os quais está fazer frente ao acirrado processo de competitividade de bens estrangeiros e tornar a economia doméstica mais eficiente e produtiva, dependem, dentre outras coisas, de uma priorização do investimento em educação. Sendo assim, é fundamental entender e quantificar o retorno desse investimento em termos de produtividade, salários e rendas domiciliares.

Nesse contexto, serão estimados os retornos dos investimentos à educação nos estados do Brasil, de acordo com o modelo desenvolvido por Mincer (1974), o qual leva em consideração o impacto da educação no nível de rendimento dos indivíduos, somando-se a isso a influência da experiência adquirida nos anos de trabalho. A opção pela abordagem de Dados em Painel, por meio de séries temporais e cross-section para os estados brasileiros, é justificada pela possibilidade de aumento significativo de observações e graus de liberdade. Ademais, como será visto, com base em Dados em Painel, pode-se testar se características individuais dos estados afetam as estimações do modelo, o que não pode ser observado caso sejam usados dados para a economia brasileira como um todo. Nesse caso, quando se identifica que efeitos individuais afetam a estimação dos regressores, o que pode ser testado pelo teste de Hausman, sugere-se que o tema em pauta não pode ser considerado como algo homogêneo entre os estados brasileiros, dando-se maior peso a elementos de economia regional.

Alguns trabalhos referentes ao tema e aplicados ao Brasil estão disponíveis na literatura e serão apresentados ao longo deste estudo, tais como Resende e Willie (2006), Salvato e Silva (2008) e Teixeira e Menezes-Filho (2012). A hipótese básica 
deste trabalho é a de que o aumento de anos de escolaridade nos estados brasileiros é acompanhado de elevação da renda domiciliar per capita, dadas algumas variáveis de controle, tais como informalidade, taxa de desemprego e experiência dos trabalhadores. Como será melhor apresentado à frente, um ponto relevante e que está como pano de fundo é o que diz respeito às estimações para as variáveis de controle de taxa de desemprego versus taxa de informalidade. Os efeitos relativos de ambas as variáveis sobre os rendimentos domiciliares podem sugerir sob diferentes ênfases relativas às políticas públicas de controle sobre o mercado de trabalho.

Por outro lado, adotando a renda domiciliar per capita como variável dependente neste trabalho, chega-se a uma medida, em nossa visão, mais representativa do retorno com educação pelos trabalhadores, que precisam fazer uso dos salários para manter um padrão de bem-estar de suas famílias, essas compostas também por indivíduos sem salários. Ainda, um caráter inovador dessa pesquisa está em sua aplicação aos dados disponíveis de todos os estados brasileiros, rompendo com o caráter ora local ora agregado na literatura existente.

O artigo está estruturado como segue: na próxima seção, serão apresentados os elementos teóricos que dizem respeito à abordagem minceriana e à importância de se considerar o fator capital humano nas discussões a respeito de desenvolvimento e crescimento econômico; a seção 3, por sua vez, traz uma breve revisão de literatura empírica sobre retornos à educação, dividida entre os resultados internacionais e o caso brasileiro; já a seção 4, apresenta as variáveis econômicas utilizadas no presente trabalho, assim como alguns elementos econométricos quanto ao método de Dados em Painel; por fim, na seção 5, analisam-se os resultados das estimações e aplica-se um sucinto teste de robustez com o intuito de dar maior confiabilidade as estimações originais. Em seguida, são feitas considerações finais e listadas as referências usadas no artigo.

\section{Capital humano e a equação minceriana}

A teoria do capital humano está relacionada com o surgimento de uma disciplina chamada Economia da Educação, nos Estados Unidos, na década de 1950. A disciplina teve origem devido à preocupação em tentar explicar os ganhos de produtividade provenientes do "fator humano", tendo como precursores Becker (1957), Mincer (1974) e Schultz (1973), professores da escola de Chicago. Alguns trabalhos mais tarde tornariam-se fundamentais nas discussões sobre crescimento econômico endógeno, tais como Lucas (1988) e Mankiw et al. (1992). A importância 
da educação no crescimento econômico moderno pode ser encontrada em um dos "novos fatos estilizados de Kaldor" (Jones; Romer, 2010), ou seja, o de que o capital humano por trabalhador está crescendo ao redor do mundo.

Fundamentado no modelo de crescimento neoclássico da década de 1950, Kaldor $(1957 ; 1961)$ teria postulado, dentre outros, o fato estilizado de que o capital físico per capita estaria crescendo em uma taxa constante. Naquele contexto, da primeira metade do Século XX, o capital humano ainda não era tema formal nas discussões e modelos que se referiam ao crescimento econômico. No entanto, hoje há evidências de que os anos de escolaridade, em crescimento ao redor do mundo, prestariam importante papel na dinâmica do PIB per capita entre os países (JONES; ROMER, op. cit.), e, portanto, na própria dinâmica do desenvolvimento econômico global.

Por outro lado, outro "novo fato estilizado de Kaldor", seria o de que, embora tenha havido ao redor do mundo uma elevação da oferta de trabalhadores com maior capital humano, não tem havido redução relativa de seus salários face aos salários de trabalhadores com menor qualificação. A explicação desse tipo de puzzle, no entanto, não é objeto deste trabalho, embora passe primeiramente pela compreensão de como os salários são determinados. Segundo Mincer (1974), o salário de um indivíduo é dado pela seguinte equação:

$$
\log W=\beta_{0}+\beta_{1} E D U C+\beta_{2} E X P+\beta_{3} E X P^{2}+Y^{\prime} x+\mu
$$

Onde, $W$ é o salário; $E D U C$ é a escolaridade; $E X P$ é a experiência; $x$ são características observáveis no indivíduo, como raça, sexo, etc; e $\mu$ é o erro amostral.

Em geral, a literatura faz uso de proxies para representar as variáveis EDUC e EXP. Na primeira, adota-se a quantidade de anos contínuos de escolaridade, ou ainda variáveis dummies para representar algum padrão discreto na educação do indivíduo; já para estimar a variável experiência, adota-se comumente a expressão:

$$
E X P=I D A D E-S-6
$$

Sendo S os anos de escolaridade e seis, por hipótese, a idade em que o indivíduo começa a estudar. Assume-se que o indivíduo começa a trabalhar após concluir seus anos de estudo.

Neste caso, os coeficientes $\beta_{1}$ e $\beta_{2}$ da equação (1) são positivos: anos a mais de escolaridade e de experiência no trabalho são acompanhados de maiores salários. Por outro lado, assume-se que a partir de certo ponto a experiência passe a representar quedas salariais por conta da redução de produtividade. Ou seja, assume-se 
rendimentos decrescentes na experiência, o que se denota por um $\beta_{3}$ negativo (SALVATO; SILVA, 2008).

É frequente o uso de outras variáveis de controle na equação (1) para se evitar vieses nas estimações devidos à omissão de variáveis ou problemas de endogeneidade (i.e. indivíduos mais ricos adquiririam mais educação). Neste trabalho, como será visto, faremos o uso de: a) taxa de desemprego e taxa de informalidade do trabalho da população dos estados brasileiros como mecanismo de correção desses possíveis vieses, uma vez que essas sejam estatisticamente significativas; b) controle da possibilidade de "causação reversa", por meio de imposição de defasagens nos regressores e teste de robustez.

\section{Breve revisão da literatura empírica}

\subsection{Resultados internacionais}

O estudo feito por Trostel et al. (2002) teve como objetivo mostrar os retornos em investimento em educação para 28 países diferentes, ${ }^{2}$ usando como referência para comparação dados obtidos entre os anos de 1985 a 1995 por intermédio da International Social Survey Programme. A amostra trabalhada foi de indivíduos empregados com idade entre 21 e 59 anos. Indivíduos que trabalham por conta própria, estudantes e aposentados não foram incluídos na pesquisa.

Embasado na teoria do capital humano e no modelo matemático de Mincer (1974) os autores estimaram a equação por meio de Mínimos Quadrados Ordinários (MQO). Foi encontrada pelos autores uma grande heterogeneidade entre as taxas de retorno. Por exemplo, enquanto na Holanda se obteve um retorno de 1,9\% para as mulheres, nas Filipinas o mesmo grupo teve retorno de 19,2\%. A explicação para essa grande diferença em retornos não foi captada pelo modelo, mas os pesquisadores notaram um padrão de que os retornos tendiam a ser mais elevados fora do continente europeu. Além disso, foi notado na pesquisa que em países com nível de educação mediano há uma tendência para a queda na renda per capita $\mathrm{e}$ nos gastos relacionados à educação.

Diferentemente dos estudos brasileiros, a renda foi mensurada por horas trabalhadas, e, sendo assim, a variável dependente foi determinada como os ganhos semanais divididos pelas horas trabalhadas. $O$ resultado geral obtido pela regressão foi de um retorno de 4,8\% para homens e de 5,7\% para as mulheres, o que de acordo com os autores, mostrou-se compatível com resultados obtidos em estudos anteriores, como, por exemplo, em Psacharopoulos (1994). 
Os autores concluíram que há uma taxa mundial média de retorno de 5\% para os homens e de aproximadamente $6 \%$ para as mulheres, sendo essa uma média global, pois de acordo com a pesquisa foi possível constatar uma grande variação nos retornos entre os países. Além disso, boa parte de sua variação cross-country no retorno à escola desafia a explicação (TROSTEL et al. 2002).

\section{$3.2 \mathrm{O}$ caso brasileiro}

Resende e Willie (2006) usaram como banco de dados a Pesquisa sobre Padrão de Vida (PPV-IBGE) de 1996 e 1997 das regiões Sudeste e Nordeste, e testaram retornos à educação pelo procedimento de dois estágios de Heckman. Os resultados obtidos na pesquisa deixaram claros os retornos para a educação formal, além de evidenciar a necessidade de um controle de qualidade para a educação.

A pesquisa publicada por Salvato e Silva (2008) abordou as principais contribuições teóricas propostas por Schultz, Becker e Mincer no que se referem aos efeitos da educação nos rendimentos de um indivíduo. A base de dados utilizada na estimação das equações de rendimentos foi a Pesquisa Nacional de Amostra por Domicílio (PNAD), do Instituto Brasileiro de Geografia e Estatística (IBGE), referente ao ano de 2005 para a região metropolitana de Belo Horizonte.

Os autores encontraram um bom ajuste aos dados, expresso no R2 ajustado de 0,36, pouco acima do resultado encontrado por Mincer (1974) para os Estados Unidos. Eles identificaram significância estatística para todos os coeficientes da regressão, além de sinais consistentes com o esperado pela teoria. $\mathrm{O}$ coeficiente associado à escolaridade foi de 0,149 , indicando que um ano a mais de escolaridade gera elevação de renda próxima de $16,15 \% .^{3}$

Um trabalho semelhante a esse foi desenvolvido para a metrópole de Porto Alegre por Bandeira e Jacinto (2010). Assim como no artigo publicado por Salvato e Silva (2008), Bandeira e Jacinto (op. cit.) consideram a educação como um dos principais componentes que irão determinar a quantidade de capital humano em uma economia. Os autores também utilizaram o banco de dados provenientes do PNAD, porém referente ao ano de 2007 . Todos os coeficientes nas equações foram significativos estatisticamente ao nível de $10 \%$. O coeficiente ligado à educação foi de 0,157 e significativo a $1 \%$, maior, portanto, que o encontrado no trabalho de Salvato e Silva (2008), enquanto o $R^{2}$ ficou em 0,33. Experiência e rendimentos decrescentes também foram significativos e com sinais esperados, ao mesmo tempo em que os autores detectaram significância estatística de variáveis como raça, sindicalização e formalização do trabalho. 
Outras pesquisas direcionadas à questão da geração de conhecimento foram feitas, porém, com um foco alternativo, a exemplo de Teixeira e Menezes-Filho (2012). A pesquisa teve como objetivo mensurar os efeitos da educação no salário do trabalhador brasileiro. Entretanto, a abordagem feita pelos autores levou em consideração o número de escolas disponíveis ao indivíduo ao nascer, população, PIB e leis que possam afetar a probabilidade de escolarização. Ou seja, os autores inseriram um caráter mais institucional à pesquisa. $\mathrm{O}$ modelo criado pelos autores também considerou a equação de rendimento desenvolvida por Mincer (1974).

O grande diferencial desta pesquisa foi a inserção do PIB dos estados, do PIB nacional e a questão institucional, que neste caso foi a lei n. 5672/1971, a lei que aglutinou os quatro anos do ensino primário com mais quatro anos de ginásio, tornando o ensino de primeiro grau de oito anos obrigatório. O trabalho corroborou a hipótese de que a lei n. 5672/1971, o número de escolas e o PIB do estado quando o indivíduo nasce tiveram um impacto positivo na escolarização da população brasileira.

\section{Dados e método}

O presente trabalho utilizou dados anuais, abrangendo o período de 1998 a 2007, para todos os estados brasileiros. Embora os dados estejam defasados, tratou-se da série mais longa disponível para o conjunto das variáveis, à época do levantamento dessa, em especial para os dados regionais de renda domiciliar per capita e anos de estudo, ambos disponibilizados pelas séries regionais do Instituto de Pesquisa Econômica Aplicada (IPEA). Em geral, as variáveis utilizadas foram renda domiciliar per capita média (W), anos de estudo (Educ), taxa de desemprego

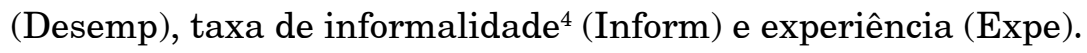

Deve ser ressaltado que a variável experiência foi obtida pelo conceito da experiência induzida. Obtém-se a experiência induzida pelo seguinte cálculo $E X P E=$ idade - escolaridade - 6 , sendo seis a idade na qual o indivíduo começa a estudar por hipótese. A idade foi obtida de uma média ponderada da expectativa de vida entre brancos e negros nos estados brasileiros, onde negros foram considerados todos aqueles não declarados brancos. Essa forma de calcular a experiência assume, de forma implícita, que o indivíduo começa a trabalhar somente após o término de seus estudos. Os dados foram coletados do Ipeadata e do Instituto Brasileiro de Estatística e Geografia (IBGE). 
Quadro 1 - Variáveis: unidades, siglas e fontes

\begin{tabular}{|l|c|c|}
\hline \multicolumn{1}{|c|}{ Variáveis } & Unidades & Siglas \\
\hline Renda domiciliar per capita - média (Proxy para renda) & $\mathrm{R} \$$ & $\mathrm{~W}$ \\
Anos de estudo - média (Proxy para escolaridade) & Anos & EDUC \\
Taxa de desemprego & $\%$ & DESEMP \\
Taxa de informalidade & $\%$ & INFORM \\
Experiência - estimativa (proxy para experiência) & Anos & EXPE \\
\hline
\end{tabular}

Fonte: dados obtidos do Ipeadata, 2013.

A renda domiciliar per capita foi deflacionada de acordo com a inflação contabilizada em outubro de 2009. Por sua vez, a variável escolaridade representa o nível de instrução que o indivíduo adquiriu durante a sua vida e é, a priori, a mais importante, pois de acordo com a hipótese deste trabalho espera-se que haja um maior impacto desta na renda. Como proxy para a escolaridade foi utilizada a média dos anos de estudo da população nos estados brasileiros, disponível no Ipeadata.

A variável taxa de desemprego mostra-nos o panorama de quantas pessoas não estão trabalhando dentro do mercado de trabalho formal, e essa variável também tem como função agir como variável de controle. A variável taxa de informalidade também age como uma variável de controle, tentando captar as pessoas que trabalham fora do mercado de trabalho formal, estatística essa disponibilizada pelo Ipeadata. Espera-se que haja correlação inversa entre a renda domiciliar e as variáveis de controle DESEMP e INFORM. Por fim, temos a variável experiência para complementar o modelo de acordo com a equação proposta por Mincer (1974). É importante ressaltar o fato de que muitos empregadores consideram experiência como um fator chave para a contratação ou não de um indivíduo. Nesse caso, espera-se que a variável EXPE tenha correlação positiva com W.

Segundo Gujarati (2006), as regressões de modelos em dados de painel geralmente tem a seguinte forma:

$$
Y_{i t}=\beta_{1}+\beta_{2} X_{2 i t}+\mu_{i t}
$$

Onde i representa a i-ésima unidade de corte transversal e o $t$ o t-ésimo período de tempo.

Quando as unidades de série $(i)$ e temporais $(t)$ tiverem o mesmo número de observações, podemos dizer que se tem um painel equilibrado. Já, por outro lado, se os números de observações diferem o painel é chamado de desequilibrado. $\mathrm{O}$ 
modelo de Efeitos Fixos tem como ideia controlar os efeitos das variáveis que foram omitidas do modelo e que podem diferir entre os indivíduos, mas constantes ao longo do tempo.

O modelo de Efeitos Fixos pode ser representado pela seguinte equação:

$$
Y_{i t}=\beta_{1 i}+\beta_{2} X_{2 i t}+\beta_{3} X_{3 i t}+\ldots+\beta_{k} X_{k i t}+\mu_{i t}
$$

onde, $\beta_{1 \mathrm{i}}$ refere-se aos coeficientes de intercepto a serem estimados para cada indivíduo.

Por outro lado, o modelo de efeito aleatório continua modelando as diferenças no comportamento dos indivíduos, mas neste caso faz com que cada unidade micro tenha um intercepto diferente, variando entre os indivíduos, mas não ao longo do tempo. O modelo de efeito aleatório é representado pela equação 5:

$$
Y_{i t}=\beta_{1 i}+\beta_{2} X_{2 i t}+\beta_{3} X_{3 i t}+\ldots+\beta_{k} X_{k i t}+\mu_{i t}
$$

Tem-se $\beta_{1 i}$, sendo aleatório e modelado da seguinte maneira:

$$
\beta_{1 i}=\beta_{1}^{*}+\mu_{i}, \operatorname{com} i=1, \ldots, n,
$$

na qual $\beta_{1}^{*}$ é um parâmetro desconhecido, representando o intercepto populacional médio; $\mu_{i}$ é o erro aleatório não observável que representa as diferenças no comportamento dos indivíduos, além disso, $\mu_{i}$ não são correlacionados com as variáveis explicativas. Unindo as equações 5 e 6 , tem-se o modelo geral de efeito aleatório que é representado pela seguinte equação:

$$
Y_{i t}=\beta_{1}^{*}+\beta_{2} X_{2 i t}+\beta_{3} X_{3 i t}+\ldots+\beta_{k} X_{k i t}+V_{i t}
$$

na qual $V_{i t}=\mu_{i t}+\mu_{i}$ ou seja, é o erro global $\mu_{i t}$ e o erro específico individual $\mu_{i}$ , e esse representa as diferenças entre os indivíduos, variando de acordo com as unidades micro e sendo constante no decorrer do tempo. Uma vez que os erros do mesmo indivíduo em distintos períodos de tempo sejam correlacionados, a técnica de MQO deixa de ser eficiente. Nesse caso, a técnica mais adequada para o modelo de efeitos aleatórios é d aos Mínimos Quadrados Generalizados (MQG). 


\section{Resultados}

O modelo com Dados Empilhados gerou resultados consistentes com o previsto pela teoria. As variáveis INFORM, EDUC e DESEMP têm efeitos significativos estatisticamente e com sinais esperados sobre o log da renda domiciliar per capita dos estados brasileiros. As variáveis de controle, INFORM e DESEMP, como esperado, tem correlação inversa com a variável dependente, enquanto a variável educação (EDUC), com correlação positiva, tem o maior impacto dentre as variáveis explicativas do modelo. De acordo com a literatura a respeito do tema, o retorno à educação pode ser calculado pela seguinte fórmula: $[\exp (\beta)-1] \times 100$, sendo $\mathrm{b} o$ coeficiente para EDUC. Logo, pode-se dizer que no modelo de Dados Empilhados o retorno à educação para os estados brasileiros é de 16,91\%. Uma maneira de interpretar esse valor seria pela sentença de que um ano a mais de estudos para a população dos estados brasileiros dá um retorno de $16,91 \%$ sobre os custos públicos com a oferta deste ano adicional de estudo. Por outro lado, a variável EXPE não teve significância estatística. A escolha entre Dados Empilhados e Dados em Painel pode ser inferida pelo Teste F aplicado ao modelo de Dados em Painel com Efeitos Fixos. Uma vez que esse rejeitou a hipótese nula, deve-se optar pelo último modelo, em detrimento ao de Dados Empilhados.

No entanto, a escolha entre Dados em Painel com Efeitos Fixos e Aleatórios requer a aplicação de um teste específico, tal como o teste de Hausman, aplicado sobre o modelo com efeitos aleatórios. Neste último, a técnica adotada foi a de MQG e não houve variação expressiva nas estimações dos coeficientes. Houve significância estatística e sinal esperado para as variáveis INFORM, EDUC e DESEMP, e a variável EXPE não apresentou significância, nem a 10\%, a exemplo do observado com Dados Empilhados. Pela fórmula do retorno à educação, caso o melhor modelo fosse o de Efeitos Aleatórios, um ano adicional de estudos daria retorno de 15,10\% aos estados brasileiros. Contudo, o teste de Hausman rejeitou a hipótese nula de não correlação entre os efeitos individuais e os regressores, de modo que se deve optar pela equação com Efeitos Fixos.

Mais uma vez, houve significância estatística e sinal esperado para INFORM, EDUC e DESEMP, e a variável EXPE não se mostrou significativa. O modelo com Efeitos Fixos apresentou melhor qualidade de ajuste aos dados, quando comparado com as duas abordagens anteriores, o que se pode inferir pelo seu maior $\mathrm{R}^{2}$ ajustado. Já o retorno à educação é reduzido para $13,45 \%$ na equação com Efeitos Fixos, mostrando que uma má especificação do modelo poderia levar a uma superestimação dos retornos à educação nos estados brasileiros. 
Deve-se ressaltar que, além de políticas públicas de elevação dos anos de escolaridade da população dos estados brasileiros, políticas estruturais de redução da informalidade podem surtir efeitos importantes sobre as rendas domiciliares per capita, assim como políticas conjunturais de redução do desemprego.

Tabela 1 - Estimações para o log da renda domiciliar per capita dos estados brasileiros pelos modelos de MQO para Dados Empilhados e Dados em Painel com Efeitos Fixos e de MQG para efeitos aleatórios: 1998 a 2007

\begin{tabular}{|c|c|c|c|c|c|c|c|c|c|}
\hline & \multicolumn{3}{|c|}{$\begin{array}{l}\text { Dados Empilhados } \\
\text { (MQO) }\end{array}$} & \multicolumn{3}{|c|}{$\begin{array}{c}\text { Painel com Efeitos Aleatórios } \\
\text { (MQG) }\end{array}$} & \multicolumn{3}{|c|}{$\begin{array}{l}\text { Painel com Efeitos Fixos } \\
\text { (MQO) }\end{array}$} \\
\hline & Coeficiente & $\sigma$ & $\mathrm{p}$-valor & Coeficiente & $\sigma$ & $\mathrm{p}$-valor & Coeficiente & $\sigma$ & $p$-valor \\
\hline C & 1,2610 & 4,1153 & 0,7596 & 6,4108 & 4,6604 & 0,1702 & 9,1453 & 5,5965 & 0,1037 \\
\hline INFORM & $-0,0158$ & 0,0016 & 0,0000 & $-0,0166$ & 0,0018 & 0,0000 & $-0,0176$ & 0,0021 & 0,0000 \\
\hline EDUC & 0,1563 & 0,0137 & 0,0000 & 0,1407 & 0,0146 & 0,0000 & 0,1262 & 0,0168 & 0,0000 \\
\hline DESEMP & $-0,0230$ & 0,0034 & 0,0000 & $-0,0161$ & 0,0034 & 0,0000 & $-0,0118$ & 0,0036 & 0,0013 \\
\hline EXPE & 0,1662 & 0,1486 & 0,2643 & $-0,0092$ & 0,1689 & 0,9568 & $-0,0967$ & 0,2036 & 0,6353 \\
\hline $\mathrm{EXPE}^{2}$ & $-0,0013$ & 0,0013 & 0,3182 & 0,0002 & 0,0015 & 0,9040 & 0,0009 & 0,0018 & 0,6211 \\
\hline$R^{2}$ ajustado & & 0,8819 & & & 0,8492 & & & 0,9149 & \\
\hline $\begin{array}{l}\text { Estatística F } \\
\text { (Prob.) }\end{array}$ & & 0,0000 & & & 0,0000 & & & 0,0000 & \\
\hline Teste de Hausman & & & & & 0,0014 & & & & \\
\hline
\end{tabular}

Nota: $\sigma$ para desvio-padrão.

Fonte: elaboração própria.

\subsection{Causação reversa e teste de robustez}

Um problema que pode estar visando os resultados das estimações realizadas é o conhecido como "causação reversa". No caso em análise, isso pode ocorrer porque é possível que haja uma endogenia na educação, ou seja, o fato de que indivíduos com maior nível de renda tenham mais acesso à educação.

Para tentar controlar essa possibilidade e testar a robustez das estimações anteriores, procederemos em uma estimação por Dados Empilhados da equação minceriana, mas adotando uma defasagem temporal nos regressores INFORM, EDUC, DESEMP, EXPE e EXPE ${ }^{2}$, de modo que se elimine a possibilidade de causação reversa contemporânea entre educação e renda. Essa regressão não tem a função de substituir as estimações da Tabela 1 para efeito de análise, mas de testar a robustez dessas, verificando se há um poder preditivo de educação sobre os rendimentos 
domiciliares. Havendo correlação com significância estatística entre educação defasada e renda domiciliar no período t, em tese, aumenta-se a chance de que as estimações da Tabela 1 representem relações sem endogenia na educação.

A análise da estimação revela que não há grandes variações no que diz respeito aos coeficientes e seus sinais, se comparada com as estimações anteriores. Mais anos de escolaridade tem poder preditivo sobre a renda domiciliar per capita dos estados brasileiros, significante estatisticamente a 1\%. Da mesma forma, as variáveis de controle INFORM e DESEMP continuam a apresentar correlações negativas e significantes com $\log (\mathrm{W})$, e a experiência permanece sem impacto significativo sobre a equação. $\mathrm{O} \mathrm{R}^{2}$ ajustado desta regressão ficou em 0,7523 e a estatística $\mathrm{F}$ rejeita a hipótese nula a $1 \%$. Neste sentido, as estimações apresentadas na Tabela 1 podem ser usadas para efeito de análise dos retornos médios à educação nos estados brasileiros de maneira mais confiável, reduzindo-se a possibilidade de viés por "causação reversa".

Tabela 2 - Equação de Dados Empilhados com lag nos regressores: teste de robustez e eliminação de causação reversa

\begin{tabular}{l|c|c|c|c}
\hline \multicolumn{1}{c|}{ Variável } & Coeficiente & $\sigma$ & Estatística t & P-valor \\
\hline C & 8.9425 & 7.6286 & 1.1722 & 0.2424 \\
INFORM(-1) & -0.0131 & 0.0029 & -4.5817 & 0.0000 \\
EDUC(-1) & 0.1254 & 0.0245 & 5.1072 & 0.0000 \\
DESEMP(-1) & -0.0128 & 0.0065 & -1.9594 & 0.0514 \\
EXPE $(-1)$ & -0.1179 & 0.2749 & -0.4289 & 0.6684 \\
EXPE $(-1)^{2}$ & 0.0013 & 0.0025 & 0.5155 & 0.6068 \\
\hline
\end{tabular}

Nota: $\sigma$ para desvio-padrão.

Fonte: elaboração própria.

\section{Considerações finais}

O presente trabalho aplicou a técnica econométrica de Dados em Painel para estudar os retornos médios à educação nos estados brasileiros. Em relação à literatura existente para o Brasil, isso já representa uma contribuição metodológica, visto que aquela é composta, sobretudo, por trabalhos ligados ao Brasil como um todo ou a cidades ou regiões metropolitanas. Por sua vez, a ideia de testar impactos dos anos de escolaridade sobre a renda domiciliar per capita, em nosso ver, também contribui metodologicamente, porque o bem-estar efetivo dos indivíduos, em geral, dependeria mais desse indicador do que dos salários individuais. 
O modelo com Dados Empilhados gerou resultados consistentes com a teoria. As variáveis INFORM, EDUC e DESEMP mostraram efeitos significativos estatisticamente e com sinais esperados sobre o log da renda domiciliar per capita dos estados do país. As variáveis de controle INFORM e DESEMP, como era esperado, tem correlação inversa com a variável dependente, enquanto a variável educação (EDUC), com correlação positiva, apresenta o maior impacto dentre as variáveis explicativas do modelo.

As estimações mostraram, com base no teste de Hausman, que a melhor modelagem seria por Dados em Painel com Efeitos Fixos. Verificou-se que os retornos médios à educação nos estados brasileiros, sobre as rendas domiciliares per capita, estão em $13,45 \%$. Ou seja, ao adquirir um ano adicional de escolaridade com base no ensino público e gratuito, ${ }^{5}$ a população de um estado obtém retorno de $13,45 \%$ em sua renda domiciliar per capita. Uma má especificação do método de regressão poderia levar à superestimação desse retorno.

Ademais, verifica-se que as variáveis de controle usadas no trabalho retem impacto esperado e significativo estatisticamente sobre o log da variável explicada, sendo que, ao que tudo indica políticas de controle da informalidade mostram-se com maior impacto quando comparadas com políticas conjunturais de controle de demanda e do desemprego. Isso sugere que ainda existe muito espaço para elevação do bem-estar nos estados por meio da redução gradual da informalidade, ainda muito grande no Brasil. Já a taxa de desemprego, nos últimos anos, parece ter menos espaço para reduções, já estando no entorno de 5\% no país como um todo e em contexto de inflação persistentemente acima da meta do Banco Central (BC).

Por fim, a presente pesquisa pode evoluir em algumas direções, tais como: a) por meio de testes de raiz unitária e cointegração para Dados em Painel, prática que ainda não é muito comum nos estudos brasileiros sobre o tema; b) para além da simples quantidade de anos de escolaridade, adoção de proxy para a qualidade da educação, que pode ser representada por desempenho escolar de alunos nos estados, ou por desempenho profissional dos professores. Entretanto, tal proxy é ainda de difícil disponibilidade por falta de séries regulares sobre o desempenho e/ ou por problemas amostrais, de modo que os trabalhos empíricos relacionados ao assunto ainda se limitam ao âmbito da quantidade ofertada e à demanda de educação; c) atualização dos dados para anos mais recentes, superando a defasagem imposta por restrição das fontes desses dados à época de seu levantamento para esta pesquisa. 


\title{
Average returns to education on brazilian states: an approach with panel data for per capita households' income
}

\begin{abstract}
This work studies the effects of the education on households' income in the Brazilian states, in per capita terms. The Pooled Data and Panel Data estimations were made with available data from 1998 to 2007, through the Mincer (1974) approach, including control variables and a proxy for labor experience. The main two evidences are: a) the increase of one additional year of education is correlated with return at $13.45 \%$ on households' per capita income of the Brazilian states; b) structural policies for controlling the labor informality seem to be more efficient than policies to control the unemployment rate, with regard to their effects on Brazilian families' income.
\end{abstract}

Keywords: Returns to education. Brazilian states. Panel data.

\section{Promedio retornos a la educación en los estados de Brasil: un enfoque con datos de panel para los rendimientos de los hogares per cápita}

\section{Resumen}

El presente trabajo tiene como objetivo evaluar el efecto de la educación en lo rendimiento de las familias brasileñas. Un panel de datos con los estados brasileños en el período 19982007 por el método Mincer (1974), con variables de control y el proxy de la experiencia laboral. Los dos resultados principales de la obra son: a) el aumento de un año adicional de escolarización se acompaña de retorno de $13,45 \%$ en el rendimiento per cápita de los hogares de los estados brasileños; b) Las políticas de control estructural de informalidad parecen ser más eficaces que las políticas de control de la tasa de desempleo, en lo que respecta a sus efectos sobre los rendimientos de las familias brasileñas.

Palabras clave: Retornos a la educación. Estados brasileños. Datos de panel. 


\section{Notas}

1 Gostaríamos de agradecer aos pareceristas anônimos da revista pelas sugestões e observações que contribuíram para a versão final do trabalho. Agradeço também a Matheus Albergaria de Magalhães pelas sugestões na fase preliminar desta pesquisa. Obviamente, quaisquer erros e omissões são de inteira responsabilidade dos autores.

2 Estados Unidos, Grã Bretanha, Alemanha Ocidental, Rússia, Noruega, Austrália, Holanda, Áustria, Polônia, Alemanha Oriental, Nova Zelândia, Itália, Irlanda, Japão, Hungria, Irlanda do Norte, Suécia, Eslovênia, Israel, República Tcheca, Bulgária, Eslováquia, Canadá, Tchecoslováquia, Espanha, Suíça, Letônia e Filipinas.

3 Conforme destacam os autores, o cálculo do retorno deve ser feito pela expressão $\left[\exp \left(\beta_{\text {educ }}\right)-1\right] x 100, \beta_{\text {educ' }}$, sendo o coeficiente para a educação.

4 -Taxa de informalidade: definição 1 - Uma das três diferentes definições do grau de informalidade oferecidas no Ipeadata com base na Pesquisa Nacional por Amostra de Domicílios (Pnad) do IBGE, esta taxa de informalidade corresponde ao resultado da seguinte divisão - (empregados sem carteira + trabalhadores por conta própria) / (trabalhadores protegidos + empregados sem carteira + trabalhadores por conta própria). Elaboração: Disoc/Ipea.

5 Obviamente que parte da população adquire mais escolaridade com ensino privado, porém neste caso seria preciso descontar da taxa de retorno calculada o fluxo de pagamentos e débitos nas rendas domiciliares, efetivamente reduzindo a taxa líquida de retorno. Portanto, por simplificação, neste trabalho consideramos a taxa de retorno com base na premissa de que o ensino público e gratuito está disponível a todos os indivíduos.

\section{Referências}

ALCANTÂRA, D. Investimento Público na Educação Representa 6,1\% do PIB, diz Governo. Disponível em: <http://noticias.terra.com.br/educacao>. Acesso em: 24 de jul. 2013.

BANDEIRA, L; JACINTO, A, P. Um estudo sobre retorno em escolaridade para a região metropolitana de Porto Alegre. XIII Encontro de Economia da Região Sul - ANPEC SUL, 2010.

BECKER, G. S. The Economics of Discrimination. Chicago: The University of Chicago Press, 1957.

GUJARATI, D. Econometria Básica. São Paulo: Makron Books. Quarta Edição, 2006.

JONES, C. I.; ROMER, P. M. The new Kaldor facts: ideas, institutions, population and human capital. American Economic Journal: Macroeconomics, v. 2, n. 1, p. 224-245, Jan. 2010.

KALDOR, N. A model of economic growth. The Economic Journal, v. 67, n. 268, p. 591-624, 1957.

KALDOR, N. Capital Accumulation and Economic Growth. In: F. A. Lutz; D. C. Hague (Eds.). The Theory of Capital, St. Martins Press, 1961. p. 177-222.

LUCAS, R. E. On the mechanics of economic development. Journal of Monetary Economics, v. 22, n. 1, p. $3-42,1988$.

MANKIW, N. G.; ROMER, D. H.; WEIL, D. N. A contribution to the empirics of economic growth. Quarterly Journal of Economics, v. 107, n. 2, p. 407-437, May 1992.

MINCER, J. Schooling, Experience and Earnings. NBER books, minc74-1, 1974. 
PSACHAROPOULOS, G. Returns to investment in education: a global update. World Development, v. 22, n. 9, p. 1325-1343, 1994.

RESENDE, M; WYLLIE, R. Retorno para educação no Brasil: evidências empíricas adicionais. Economia Aplicada, São Paulo, v. 10, n. 3, p. 349-365, jul.-set. 2006.

SALVATO, M; SILVA, D. O impacto da educação nos rendimentos do trabalhador: uma análise para região metropolitana de belo horizonte. In: SEMINÁRIO SOBRE A ECONOMIA MINEIRA, XIII, Anais... [Proceedings of the 13th Seminar on the Economy of Minas Gerais], 2008.

SCHULTZ, T. W. O capital humano: investimentos em educação e pesquisa. Tradução Marco Aurélio de Moura Matos. Rio de Janeiro: Zahar, 1973.

TEIXEIRA, W. M; MENEZES-FILHO, A. Estimando o retorno à educação do Brasil considerando a legislação educacional brasileira como um instrumento. Revista de Economia Política, . 32, n. 3, p. 479-496, 2012.

TROSTEL P.; WALKER, I.; WOOLLEY, P. Estimates of the economic return to schooling for 28 countries. Labour Economics, 9, p. 1-16, 2002. 